\title{
Ultrasound-enhanced ocular delivery of dexamethasone sodium phosphate: an in vivo study
}

Marjan Nabili ${ }^{*}$, Aditi Shenoy ${ }^{1}$ Shawn Chawla', Sankaranarayana Mahesh², Ji Liu², Craig Geist ${ }^{2}$ and Vesna Zderic ${ }^{1}$

\begin{abstract}
Background: The eye's unique anatomy and its physiological and anatomical barriers can limit effective drug delivery into the eye.

Methods: An in vivo study was designed to determine the effectiveness and safety of ultrasound application in enhancing drug delivery in a rabbit model. Permeability of a steroid ophthalmic drug, dexamethasone sodium phosphate, was investigated in ultrasound- and sham-treated cases. For this study, an eye cup filled with dexamethasone sodium phosphate was placed on the cornea. Ultrasound was applied at intensity of $0.8 \mathrm{~W} / \mathrm{cm}^{2}$ and frequency of 400 or $600 \mathrm{kHz}$ for $5 \mathrm{~min}$. The drug concentration in aqueous humor samples, collected $90 \mathrm{~min}$ after the treatment, was determined using chromatography methods. Light microscopy observations were done to determine the structural changes in the cornea as a result of ultrasound application.

Results: An increase in drug concentration in aqueous humor samples of 2.8 times $(p<0.05)$ with ultrasound application at $400 \mathrm{kHz}$ and 2.4 times $(p<0.01)$ with ultrasound application at $600 \mathrm{kHz}$ was observed as compared to sham-treated samples. Histological analysis showed that the structural changes in the corneas exposed to ultrasound predominantly consisted of minor epithelial disorganization.

Conclusions: Ultrasound application enhanced the delivery of an anti-inflammatory ocular drug, dexamethasone sodium phosphate, through the cornea in vivo. Ultrasound-enhanced ocular drug delivery appears to be a promising area of research with a potential future application in a clinical setting.
\end{abstract}

Keywords: Therapeutic ultrasound, Drug delivery, Cornea, Ocular diseases, Sonophoresis, Dexamethasone sodium phosphate

\section{Background}

The objective of this study was to investigate the role of ultrasound in enhancing delivery of an ocular antiinflammatory drug, dexamethasone sodium phosphate, through the cornea in a rabbit eye model in vivo. Ultrasound has been used in ophthalmology for decades but mostly as a diagnostic imaging tool [1]. For example, ultrasound biomicroscopy imaging can provide highresolution imaging of the anterior part of the eye [2]. Therapeutic ultrasound also has a potential for clinical applications in ophthalmology [3]. High-intensity focused

\footnotetext{
* Correspondence: mnabili@gwmail.gwu.edu

'Department of Electrical and Computer Engineering, George Washington University, Washington, DC 20052, USA

Full list of author information is available at the end of the article
}

ultrasound (HIFU) has been reported as an effective treatment method for reducing intraocular pressure, which showed promising results in both animal and clinical studies as a potential novel approach for glaucoma treatment [4-6]. Sonoda et al. [7] used ultrasound in conjunction with commercially available microbubbles to enhance gene delivery into the back of the eye of New Zealand albino rabbits without any ocular tissue damage observed and with 1.5 to 2 times increase in the delivery efficiency. The application of therapeutic ultrasound at frequency of $690 \mathrm{kHz}$ has also been observed as effective in moderately disrupting the integrity of the blood retinal barrier when applied as $10 \mathrm{~ms}$ bursts of $1 \mathrm{~Hz}$ for $60 \mathrm{~s}$ at pressures of up to $1.1 \mathrm{MPa}$, thus increasing the penetration of systemically administered drugs into the retina [8]. 
The eye's distinct anatomy and physiology pose challenges to the development of effective ocular drug delivery systems [9] since its various defense mechanisms make it very difficult to achieve sufficient drug penetration into the eye $[3,10]$. Topical administration of drugs into the eye is convenient, common, and well accepted by patients $[9,11]$. However, this method is highly inefficient because only $1 \%-5 \%$ of the applied ocular drug penetrate to the desired ocular site $[11,12]$. Most of the topically administrated drugs are washed away by factors such as normal tear volume, blinking, induced lacrimation [12], and nasolachrymal drainage $[9,11,12]$. Moreover, drugs can enter systemic circulation through conjunctival blood capillaries and lymphatics before reaching intraocular tissues $[12,13]$. The cornea is considered to be the main pathway for topical drug delivery [3,10]; however, the passage of ocular drugs is limited by corneal barriers [9-11]. This highly selective barrier consists of three primary layers: namely, the epithelium, which is impermeable to hydrophilic drugs because of its lipophilic properties and tight junctions; the stroma, which is hydrophilic in nature, making it a dominant barrier for lipophilic drugs; and the endothelium [14,15]. Intravitreal and periocular injections are some of the invasive methods of ocular drug delivery, which are used to avoid inefficient topical and systemic processes. However, these methods have their own side effects including infection and cataract formation [3]. Many other methods such as hydrogel contact lenses, mucoadhesive and viscosity-enhancing polymers, iontophoresis, liposomes, and nanoparticles have been used to increase concentration of the drugs delivered into the eye $[12,13]$. For example, hydrogel contact lenses can increase the bioavailability of drugs by prolonging the drug exposure time. However, these contact lenses can cause blurring of vision and local corneal tissue toxicity [16,17]. Ocular iontophoresis, while effective in delivering drugs, requires specialized drug formulations and was shown to have mild adverse effects [18]. This noninvasive technique involves applying a small electric current to enhance the penetration of ionized drug into the eye [9] and has been investigated for delivering different types of ocular drugs such as antibiotics [19] and anti-viral drugs [20]. Injectable biodegradable implants in the forms of rods, plugs, discs, or sheets have also been proposed for ocular drug delivery; however, this approach is expected to have low patient compliance due to its invasive nature [21].

The design of our study focused on promoting topical drug penetration through the cornea via ultrasound methods, potentially providing a way for topical ophthalmic drugs to reach different areas in the eye more effectively. Our approach may allow for better targeting of diseased eye tissues as compared to systemic methods, leading to usage of smaller quantities of the drug to get the desired therapeutic effect and to reduce toxicity of the treatment. In addition to delivery of commercially available ophthalmic drugs, other compounds such as macromolecules that may assist with wound repair have the potential to be coupled with ultrasound-mediated delivery [22]. Wound repair in the body is normally dependent on the presence of vasculature for the delivery of nutrients to damaged tissue; however, many of the eye's translucent structures are avascular (e.g., cornea, lens, vitreous). When damaged, these tissues do not have access to thrombospondin 1, a glycoprotein central to tissue repair, and as a result fibrosis and repair are slow, causing scarring of eye tissue and thus blindness [23].

Results from our previously performed in vivo studies showed that using $880-\mathrm{kHz}$ ultrasound at intensities of $0.19,0.34$, and $0.56 \mathrm{~W} / \mathrm{cm}^{2}$ (in a continues mode) with an exposure duration of 5 min resulted in 2.4, 3.8, and 10.6 times increases in corneal permeability respectively for a drug mimicking dye sodium fluorescein [24]. Similar results were also reported by Nuritdinov [25] who showed that ultrasound at frequencies of $470-880 \mathrm{kHz}$ and intensity of $0.3 \mathrm{~W} / \mathrm{cm}^{2}$ applied for 5 min produced up to ten times increase in the corneal permeability for sodium fluorescein in a rabbit model, in vivo.

The main goal in the study reported here was to investigate the corneal permeability for a clinically relevant anti-inflammatory drug, dexamethasone sodium phosphate, in an in vivo model, which can give us a better understating of an actual clinical situation. After investigating the effects of ultrasound application on the delivery of dexamethasone sodium phosphate in vitro [26], we decided to focus on the ultrasound parameters found optimal in these in vitro studies and utilize them in our in vivo studies.

\section{Methods}

A total of 20 healthy New Zealand white rabbits, 1-2 years old, were used in our studies. Rabbit eyes have been used as the standard model for ophthalmic drug delivery research $[14,27,28]$. All procedures were performed in accordance with Institutional Animal Care and Use Committee (IACUC) regulations under the approved protocol. The entire experiment was completed in the animal facility at the George Washington University in the span of two nonconsecutive days. An attending veterinarian assisted our research team with anesthesia and animal handling procedures. Rabbits were anesthetized with injection of ketamine $(50 \mathrm{mg} / \mathrm{kg})$ and xylazine $(5 \mathrm{mg} / \mathrm{kg})$. Anesthesia was reapplied as needed as determined by the veterinarian.

\section{In vivo experiment}

The rabbits were randomly assigned to three groups: ultrasound treatment at $400 \mathrm{kHz}(n=6)$, ultrasound treatment at $600 \mathrm{kHz}(n=6)$, and sham treatment group 
$(n=8)$. The sham treatment experiments were set up exactly the same as ultrasound-treated experiments. Ultrasound transducer was submerged inside the solution in eye cup, and all of the procedures were the same (except that the ultrasound power button was not turned on). In all cases, only one of the rabbit's eyes received the treatment. The other eye was used as a control where no ultrasound application or drug exposure was present. These control eyes were used for histological observations. The ophthalmic drug used in our experiments was dexamethasone sodium phosphate, which had also been used during our previous in vitro studies [26]. Dexamethasone sodium phosphate is used in ophthalmology as a $0.1 \%$ topical steroid solution (Bausch \& Lomb Inc, Tampa, FL, USA) applied in the treatment of inflammatory conditions. It has a molecular weight of $516.41 \mathrm{Da}$ and hydrophilic properties.

Unfocused circular ultrasound transducers (Sonic Concepts, Bothell, WA, USA) with 15-mm active diameters were used in the experiments and were positioned at near to far field transition distance from the eye to ensure optimal energy delivery. The transition point from the near field to the far field, $d_{\mathrm{ff}}$, is the location of the furthest maximum pressure for the unfocused transducer [29]. These transducers operated at frequencies of 400 and $600 \mathrm{kHz}$, and the $d_{\mathrm{ff}}$ calculated for each transducer frequency was 1.5 and $2.25 \mathrm{~cm}$, respectively. The driving unit consisted of a function generator (33250, Agilent, Santa Clara, CA, USA) connected to the power amplifier (150A100B, Amplifier Research, Souderton, PA, USA), which was connected to the ultrasound transducer via an electrical power meter (Sonic Concepts, Bothell, WA, USA).

The eye cup was designed in such a way that it could hold up to $25 \mathrm{~mL}$ of solution by adding an extension above the standard eye cup area as shown in Figure 1. This extension was added to ensure that the transducers would be submerged in the drug solution while still held within the $d_{\text {ff }}$ distance from the corneal surface. A metal xstand was placed inside the eye cup to secure the

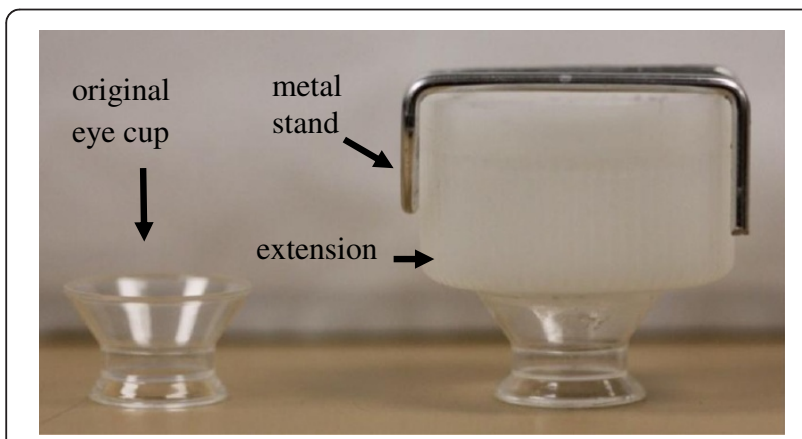

Figure 1 Eye cup design. Original and modified eye cup used in in vivo experiments. transducers in place and keep them at $d_{\mathrm{ff}}$ distance from surface of the cornea during ultrasound and sham treatments. Ultrasound transducer was submerged in the drug solution inside the eye cup during the experiment.

During treatment, therapeutic ultrasound was applied at the parameters that were shown most effective in our previous in vitro studies. The in vitro results specifically showed that using lower frequency ultrasound (in the $400 \mathrm{kHz}$ to $1 \mathrm{MHz}$ range) was more effective in increasing the permeability of the cornea [26]. The ultrasound intensity used in the in vivo studies was $0.8 \mathrm{~W} / \mathrm{cm}^{2}$, and duration of ultrasound application was $5 \mathrm{~min}$. The ultrasound transducers and the eye cup were rinsed with saline before procedure. The eye cup (Figure 2) was placed under the rabbit eyelid and filled with the drug solution after the animal was anesthetized. Ultrasound application started immediately after the eye cup was placed on the eye and filled with the drug solution. The transducer with the active diameter of $15 \mathrm{~mm}$ covered the entire surface of the cornea which had a diameter of 14-15 mm. After $5 \mathrm{~min}$, ultrasound application was stopped and the transducer and the drug solution were removed from the eye cup, followed by a careful removal of the eye cup from the animal. The eye was not rinsed afterwards.

The solution was kept at room temperature (approximately $25^{\circ} \mathrm{C}$ ) before the starting of the experiments. The temperature of drug solution inside the eye cup in the proximity of the cornea was measured (Figure 3) during ultrasound application using a thermometer (dual thermometer, Fisher Scientific, Atlanta, GA, USA). The thermocouple used was a $\mathrm{K}$ type with approximately 1-mm width. The thermometer was not touching the cornea to prevent accidental damage. Temperature was recorded at initial $(t=0 \mathrm{~min})$, midpoint $(t=2.5 \mathrm{~min})$, and final $(t=5 \mathrm{~min})$ times after the start of ultrasound application.

After ultrasound application, in vivo gross observation of the cornea was performed using a high-magnification stereomicroscope. This procedure was repeated after 80 min from the start of the experiment, before the animal was euthanized.

At approximately $90 \mathrm{~min}$ after the ultrasound treatment, the animal was euthanized (using Euthasol at $1 \mathrm{~mL} / 4.54 \mathrm{~kg}$ ) and $0.3 \mathrm{~mL}$ sample of the aqueous humor was collected subsequently using a $27 \mathrm{G} \times 1 / 2$ in. needle (12.7 mm length). The aqueous humor samples were stored in a freezer at $-80^{\circ} \mathrm{C}$. Once the aqueous humor was sampled, the eyes were excised and the cornea was dissected and fixed in formalin (Protocol ${ }^{\circledR}$, Fisher Scientific Company, Kalamazoo, MI, USA).

\section{Histology}

The cornea samples were prepared using hematoxylin and eosin ( $\mathrm{H} \& \mathrm{E})$ staining procedures for histology slides (Histoserv, Inc., Germantown, MD, USA). In our 


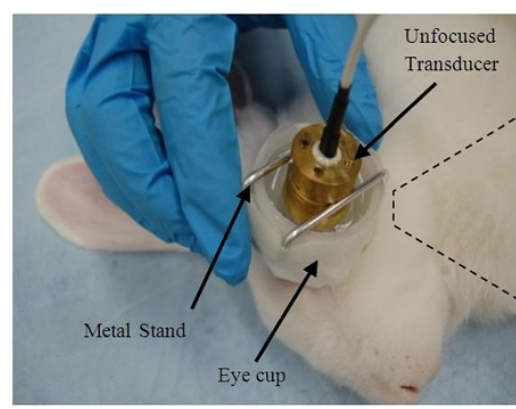

$\mathbf{a}$

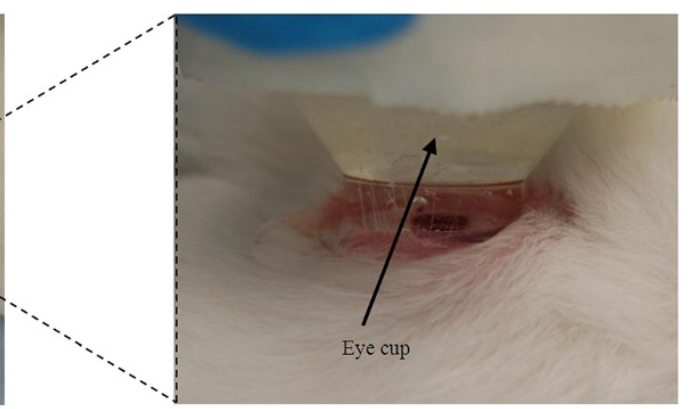

b

Figure 2 In vivo setup. (a) The eye cup and transducer placement. (b) Close-up of the eye cup placement on the rabbit eye in vivo.

previous in vitro studies [26], we developed a procedure for the determination of changes in the epithelial layer of the cornea, where corneas were classified into four groups based on the level of epithelial damage. Each class was given a value of $0,1 / 3,2 / 3$, or 1 (please see Figure 2 from Nabili et al. [26]). Class 0 (value of 0 ) has no epithelial disorganization (all cells appear intact; the layers of epithelium are well organized). Class 1 (value of 1/3) has minor epithelial disorganization (some of the cells appear necrotic; some cells are missing or the first layer of epithelium is removed). For class 2 (value of 2/3), a more severe epithelial disorganization is observed (more cells appear necrotic or missing; cells in the two to three layers of epithelium appear damaged). Class 3 (value of 1 ) has the most severe epithelial disorganization (majority of the cells are ruptured; all of the epithelial layers are absent or severely damaged).

The thickness of the different layers of the cornea (epithelium, stroma, and endothelium) was also determined from the histological slides. To determine the thickness, measurements of the width of each layer, were performed at the same three locations on each sample. Each cornea sample was examined using Zeiss AxioImager light microscope (Carl Zeiss, Jena, Germany) at × 5-20 magnification. The three locations that were measured on each cornea sample were at the center of the cornea (location 1),

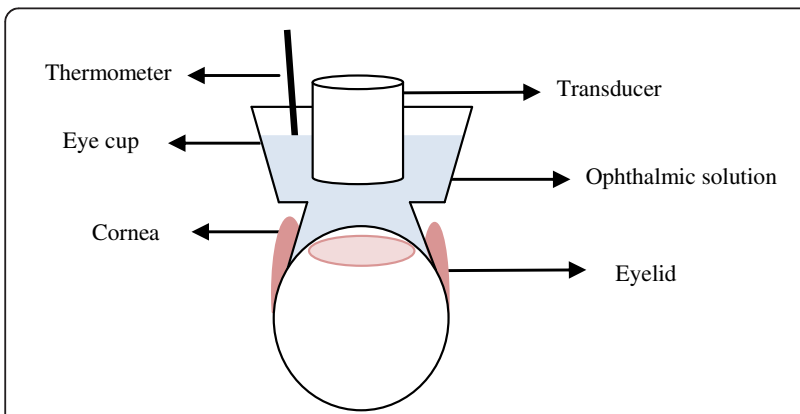

Figure 3 Schematic of in vivo setup. Placement of the thermocouple and transducer with respect to the eye. midway between the left end of the cornea and the center (location 2), and midway between the right end of the cornea and the center (location 3). These measurements were reported in micron. If any of the corneal layers were missing, they were recorded as 'absent' and were shown in calculations as a ' 0 ' value.

\section{Chromatography}

Aqueous humor samples were sent to a facility (Cayman Chemical Co., Ann Arbor, MI, USA), which used chromatography methods to investigate the amount of dexamethasone sodium phosphate inside sham- and ultrasound-treated samples. A calibration curve for dexamethasone sodium phosphate was first generated, followed by the determination of the amount of dexamethasone sodium phosphate in aqueous humor samples. Two out of twenty samples sent to the facility were not analyzed due to inadequate amounts of aqueous humor in these samples.

Based on the protocol provided by this chromatography facility, the procedure to generate calibration curves for dexamethasone and measuring amount of dexamethasone in an aqueous humor sample is explained as follows: to create the calibration curve for dexamethasone, $10 \mu \mathrm{L}$ of dexamethsone calibration standard solution $(100 \mu \mathrm{g} / \mathrm{mL})$ was added to a microcentrifuge tube with $990 \mu \mathrm{L}$ of 95:5 $(v / v)$ water/acetonitrile, vortex mixed for about $15 \mathrm{~s}$, and subsequent $500 \mu \mathrm{L}$ serial dilutions were made. Fifty microliter of dexamethasone-d4 internal standard solution was added to $100 \mu \mathrm{L}$ of each standard and vortexed for approximately $15 \mathrm{~s} ; 1 \mathrm{~mL}$ of methyl tert-butyl ether (MTBE) was added to each and vortexed for about $15 \mathrm{~s}$ and then centrifuged for $10 \mathrm{~min}$. The organic layer was transferred to a $1.5-\mathrm{mL}$ auto-sampler vial and dried under nitrogen and reconstituted with $100 \mu \mathrm{L}$ of 95:5 $(v / v)$ water/ acetonitrile. To investigate the amount of dexamethasone inside the aqueous humor sample from our experiments, $10 \mu \mathrm{L}$ of sample with unknown concentration of dexamethasone sodium phosphate and $90 \mu \mathrm{L}$ of water were added to a microcentrifuge tube, and $50 \mu \mathrm{L}$ of dexamethasone- $\mathrm{d} 4$ internal standard solution was added 
to one $100 \mu \mathrm{L}$ of each standard and vortexed for about $15 \mathrm{~s}$. One milliliter of MTBE was added to 46 each, vortexed about $15 \mathrm{~s}$, and centrifuged for $10 \mathrm{~min}$. The organic layer was transferred to a $1.5-\mathrm{mL}$ auto-sampler vial and dried under nitrogen and reconstituted with $100 \mu \mathrm{L}$ of 95:5 (v/v) water/acetonitrile.

\section{Results}

The statistical test used to analyze the data was a $t$-test: two-sample assuming unequal variances. Figure 4 shows the comparison between the dexamethasone sodium phosphate concentration in aqueous humor in ultrasoundtreated and sham-treated samples. The increase in drug concentration in aqueous humor samples was 2.8 times $(p<0.05)$ at ultrasound frequency of $400 \mathrm{kHz}(n=5)$ and 2.4 times $(p<0.01)$ at frequency of $600 \mathrm{kHz}(n=6)$, when compared to sham-treated samples $(n=7)$. Drug concentration increase in the eye in case of sham-treated samples was $593.8 \pm 351.9 \mathrm{ng} / \mathrm{mL}$. This value was $1,658.5 \pm 823 \mathrm{ng} /$ $\mathrm{mL}$ using $400-\mathrm{kHz}$ frequency and was $1,453.8 \pm 434.3 \mathrm{ng} /$ $\mathrm{mL}$ using $600-\mathrm{kHz}$-frequency ultrasound.

No gross damage of the cornea was detected using stereomicroscopy observations immediately after the treatment and $80 \mathrm{~min}$ after the treatment. In subsequent histological observations, ultrasound-induced changes were observed in the epithelial layer of the cornea including missing cells and in some cases detachment of whole epithelial cell layers. No apparent changes were observed in the stroma and endothelium; however, detachment of the endothelium was observed in some sham-treated and ultrasound-treated samples and may be caused by processing artifacts [30,31]. Representative light microscopy images of control, sham-treated, and ultrasound-treated corneas are shown in Figure 5. Results of histological analysis of ultrasound- and sham-treated corneas based on the four classes of epithelial damage, as described in the 'Methods' section, are shown in Figure 6. The increase in the epithelial damage was four times $(p<0.01)$ at ultrasound frequency of $400 \mathrm{kHz}(n=6)$ and three times $(p<0.05)$ at frequency of $600 \mathrm{kHz}(n=6)$, as compared to sham-treated samples $(n=8)$.

The quantitative changes in the corneal structure was $0.25 \pm 0.46$ in sham-treated samples, $1.7 \pm 0.51$ using $400-\mathrm{kHz}$ frequency, and $1.0 \pm 0.63$ using $600-\mathrm{kHz}-$ frequency ultrasound. These values are calculated based on the characteristic model designed to analyze corneal damage in semi-quantitative matter (see 'Methods' section).

Despite the fact that aqueous humor samples from all eyes were sent to a chromatography facility, the amount of drug in one sample from $400-\mathrm{kHz}$ batch and one from sham-treated batch was not investigated due to inadequate amount of samples for the equipment. However, all cornea samples which were sent to a histological facility were processed. For these reasons, there is a difference between samples of aqueous humor and cornea samples used for histology analysis.

Figure 7 shows thickness of different layers of cornea in ultrasound- and sham-treated samples. The mean thickness of ultrasound-treated epithelium was smaller as compared to control and sham-treated cases. For $400-\mathrm{kHz}$ ultrasound exposure, changes in the epithelial thickness were observed as $57 \%(p<0.001)$ decrease as compared to control epithelium and $49 \%$ decrease as compared to sham-treated samples (Figure 7a). For $600-\mathrm{kHz}$ ultrasound treatments, apparent decrease in the epithelial thickness was $33 \%$ as compared to control samples and $19 \%$ as compared to sham-treated samples (with no statistical significance) (Figure 7a). The thickness of the ultrasound-

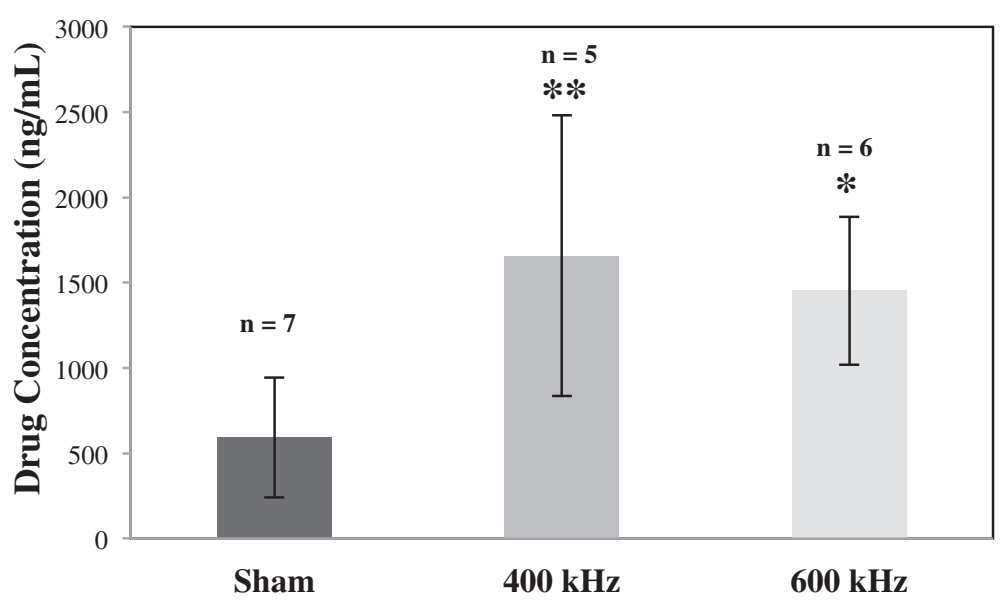

Figure 4 Dexamethasone sodium phosphate concentration in aqueous humor. Dexamethasone sodium phosphate concentration increased in aqueous humor as a result of ultrasound application as compared to sham-treated samples $(n=7)$. Ultrasound was applied at frequencies of $400 \mathrm{kHz}(n=5)$ and $600 \mathrm{kHz}(n=6)$, intensity of $0.8 \mathrm{~W} / \mathrm{cm}^{2}$, and exposure duration of $5 \mathrm{~min}$. Data are given as mean \pm standard deviation. ${ }^{*} p$ value $<0.05,{ }^{* *} p$ value $<0.01$. 

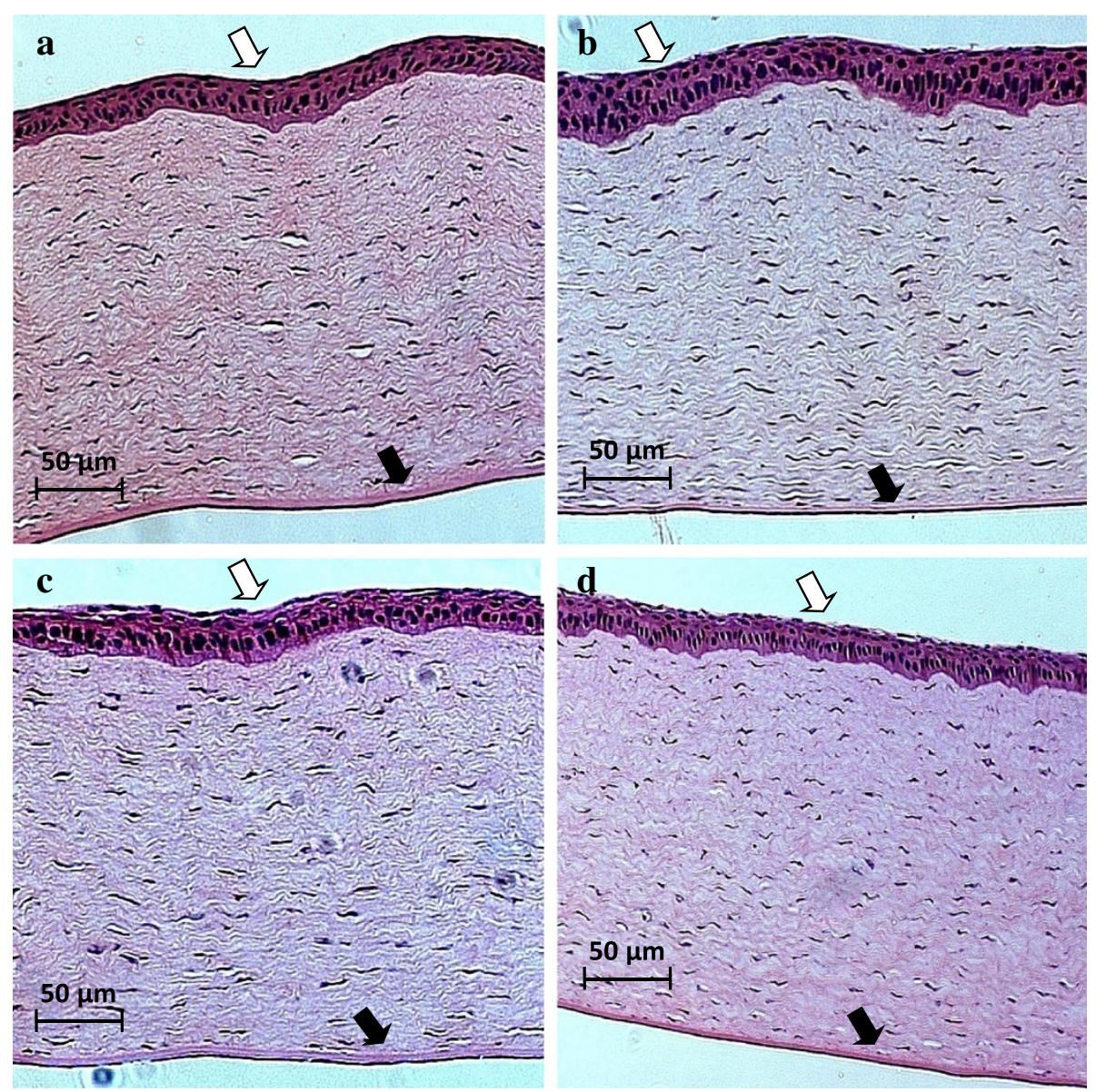

Figure 5 Histology analysis. (b) A control cornea, (b) a sham-treated cornea, (c) a corneal sample exposed to 400-kHz ultrasound, and (d) a corneal sample exposed to $600-\mathrm{kHz}$ ultrasound. The epithelium is the eosinophilic layer, indicated by white arrow, as opposed to much thinner endothelium, which is just a cell layer in thickness, marked with a black arrow. Magnification of $\times 10$ was used.

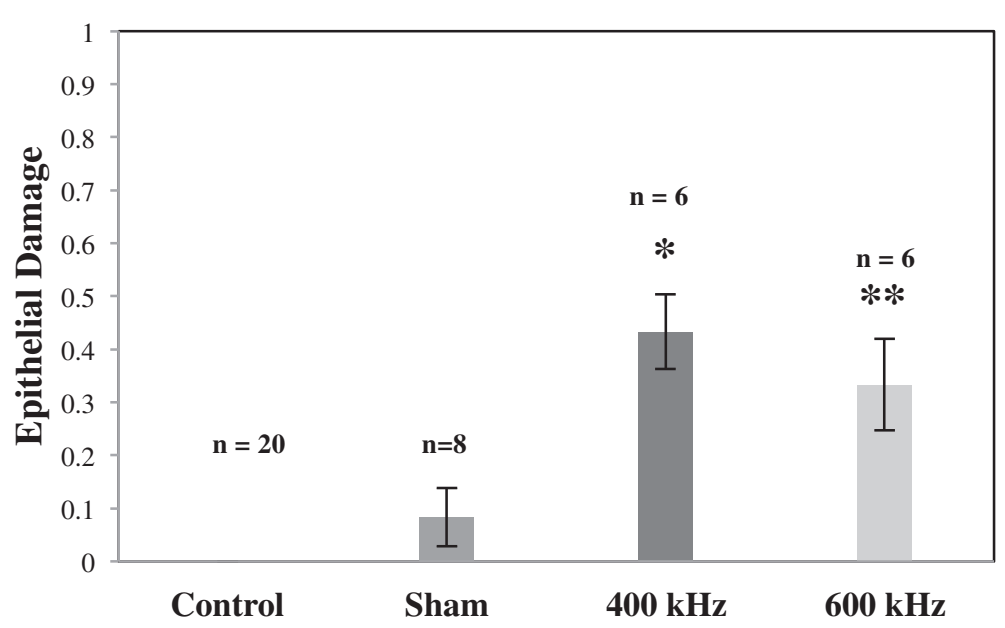

Figure 6 Change in the corneal structure. Changes in the cornea as a result of ultrasound application as compared to sham-treated corneas $(n=8)$. Different bars represent the corneal damage due to ultrasound application at frequencies of $400 \mathrm{kHz}(n=6)$ and $600 \mathrm{kHz}(n=6)$. Data are shown as mean \pm standard deviation. No changes were observed in control corneas $(n=20)$ in all cases. ${ }^{*} p$ value $<0.05 ;{ }^{* *} p$ value $<0.001$. 

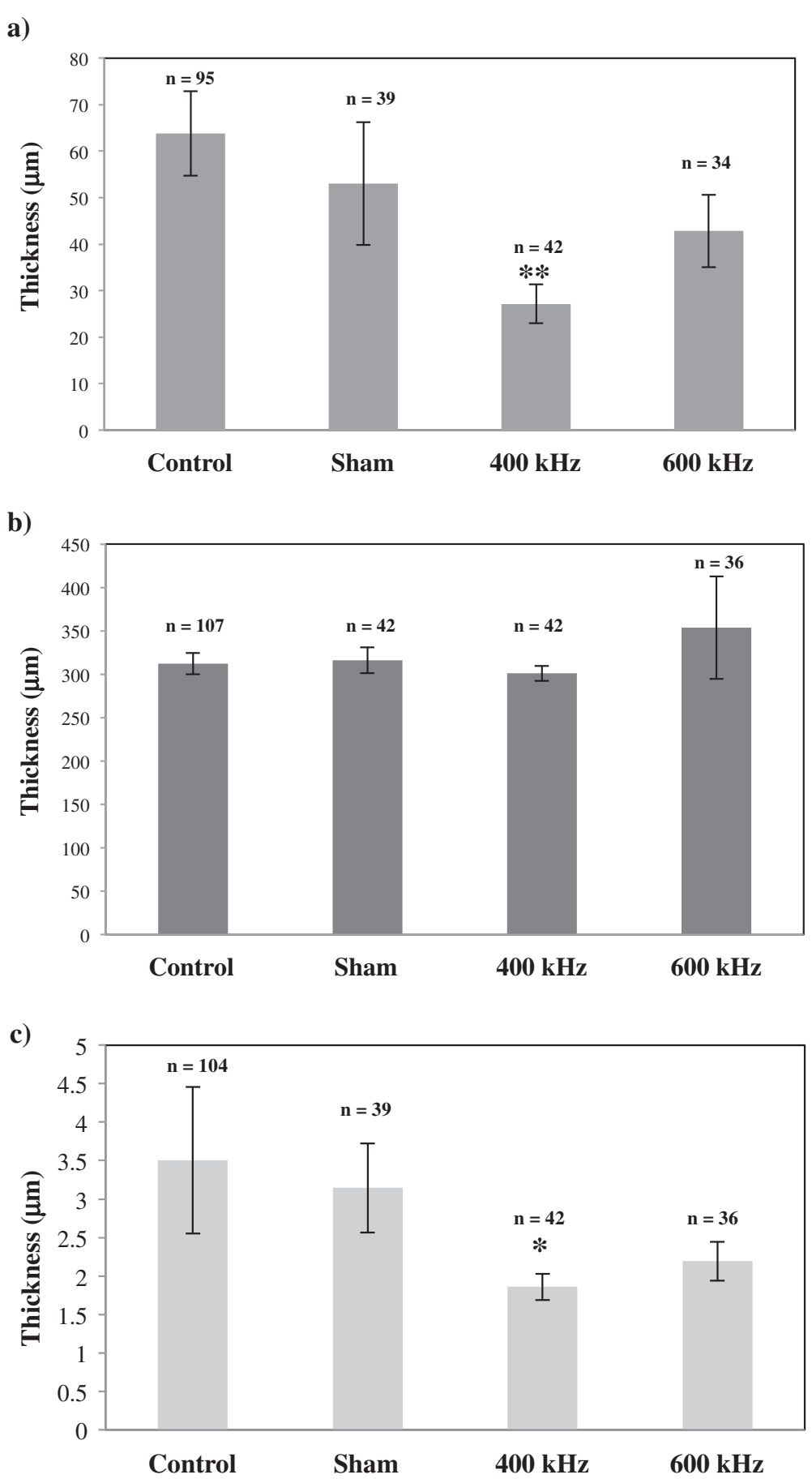

Figure 7 Thickness of different layers of the cornea. This graph shows thickness of the different layers of the cornea: (a) epithelium, (b) stroma, and (c) endothelium. Different bars represent results for control, sham-treated, 400- and 600-kHz-ultrasound-treated samples. ${ }^{*} p$ value $<0.05$ as compared to sham values; ${ }^{* *} p$ value $<0.001$ as compared to control values.

treated stroma was not significantly different from control and sham-treated stroma (Figure 7b). Endothelial thickness appeared to decrease to $10 \%$ for sham-treated samples as compared to controls with no statistical significance. Comparison between sham-treated and ultrasound-treated endothelium showed $41 \%$ decrease $(p<0.05)$ and 30\% decrease for 400 and $600 \mathrm{kHz}$, respectively (Figure 7c).

Figure 8 shows the relation between drug concentration in the aqueous humor and the corneal damage as calculated based on the four classes of epithelial damage 


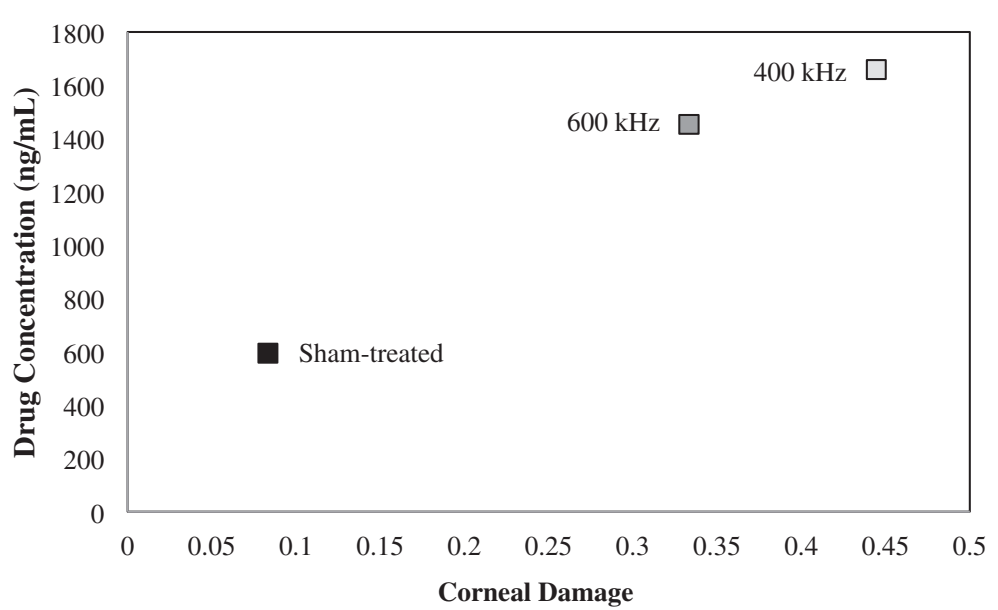

Figure 8 Relation between drug concentration in aqueous humor and corneal damage. This figure shows a relationship between corneal damage and drug concentration in the aqueous humor for ultrasound- and sham-treated cases. Data are given as mean \pm standard deviation.

(as shown in Figure 6). There appears to be a direct relation between the drug concentration increase and the level of corneal damage.

The change in temperature from $t=0$ to $t=5 \mathrm{~min}$ for sham-treated cases was $0^{\circ} \mathrm{C}-3^{\circ} \mathrm{C}$ (mean \pm standard deviation of $1.4^{\circ} \mathrm{C} \pm 1.2^{\circ} \mathrm{C}$ ). In ultrasound-treated cases, the change of temperature for the same period of time was $3^{\circ} \mathrm{C}-6^{\circ} \mathrm{C}$ (mean \pm standard deviation of $4.0^{\circ} \mathrm{C} \pm 1.1^{\circ} \mathrm{C}$ ) for $400-\mathrm{kHz}$ ultrasound and $4^{\circ} \mathrm{C}-5^{\circ} \mathrm{C}$ (mean \pm standard deviation of $4.8^{\circ} \mathrm{C} \pm 0.4^{\circ} \mathrm{C}$ ) for $600-\mathrm{kHz}$ ultrasound.

\section{Discussion}

The potential advantages of using ultrasound for drug, gene, and protein delivery, using parameters that cause cavitation and streaming effects, have been confirmed in different experimental studies $[24,28,32]$. Cavitation is known as the creation, oscillation, and collapse of gas bubbles due to acoustic waves [33-35]. It is stated to be the main mechanism responsible for promoting drug delivery through the skin [32,36]. There are two types of cavitation: stable and inertial. Stable cavitation is defined as the uniform pulsation of bubbles over long time intervals [33], and the oscillation of these bubbles can produce mechanical stresses that may cause cell membrane rupture [37]. In the case of inertial cavitation, collapse of the bubbles causes shock waves and microjets to be generated near the cells, thus producing pits in the cell membranes $[38,39]$. The effect of inertial cavitation is greater at lower frequencies because bubbles have more time to grow, which results in a more violent collapse [40]. Based on our previous studies [24], at ultrasound frequency of $880 \mathrm{kHz}$, cavitation activity was shown to correlate with enhancement of drug delivery through the cornea in vivo, and similar mechanisms are expected here. The mechanism, which has an effect on increasing the drug penetration through the cornea, is thought to be cavitation and generation of bubbles. In this study, stable cavitation appeared to be the cause of corneal permeability enhancement in the case of ultrasound applied at $0.19 \mathrm{~W} / \mathrm{cm}^{2}$, however; both stable and inertial cavitation were present when higher intensities of $0.34-0.56 \mathrm{~W} / \mathrm{cm}^{2}$ were used [24].

There is an inverse relationship between ultrasound frequency and cavitation effects. It is difficult to generate cavitation at higher frequency because the time between the positive and negative acoustic pressure is short [41]. As a result, the gas that is dissolved in a medium does not have the ability to diffuse into the cavitation nuclei [41]. For the purpose of this study, we chose ultrasound frequencies of 400 and $600 \mathrm{kHz}$ which belong to medium frequency range; therefore, cavitation was one of the likely mechanisms that cause an increase in corneal permeability. Results from our in vitro study for dexamethasone sodium phosphate showed that corneal permeability had a statistically significant increase of $43 \%$ to $109 \%$ after ultrasound exposure at $400 \mathrm{kHz}$ (at intensity of 0.3 to $1.0 \mathrm{~W} / \mathrm{cm}^{2}$ applied for $5 \mathrm{~min}$ ) as compared to shamtreated samples. The increase in corneal permeability observed in case of $400-\mathrm{kHz}$ ultrasound was more effective as compared to ultrasound application at $600 \mathrm{kHz}$ (46\%-55\% permeability increase at intensities of 0.3 to $\left.1.0 \mathrm{~W} / \mathrm{cm}^{2}\right), 800 \mathrm{kHz}(50 \%-72 \%$ increase at intensities of 0.5 to $\left.1.0 \mathrm{~W} / \mathrm{cm}^{2}\right)$, and $1 \mathrm{MHz}(46 \%-63 \%$ increase at intensities of 0.5 and $0.8 \mathrm{~W} / \mathrm{cm}^{2}$ ).

In addition to cavitation effects, streaming and microstreaming may have played a role in increasing corneal permeability. Acoustic streaming is identified as a force, which is able to move ions and small molecules as a result of physical action of ultrasound waves [42]. Shearing and streaming forces associated with ultrasound may disrupt cell walls, internal organelles, and tissues if applied at high enough intensities [29]. Further, acoustic 
streaming was recognized to be an important factor in the enhancement of drug delivery through the skin $[32,36]$. When bubbles oscillate in an ultrasound field, a microscale circulatory motion forms around the oscillating bubble and is called microstreaming [43,44]. The shear stresses generated by microstreaming near cell boundaries, due to expansion and contraction of microbubbles, may cause cell membrane rupture [37] and result in hemolysis, release of protein from bacteria, and mechanical disruption of plant cells [37,45]. Further, microstreaming was shown to cause an increase in the movement of blood and fluids around the thrombus and enhance drug infusion into the thrombus [46]. Therefore, streaming and microstreaming are important ultrasound mechanisms that have the potential to increase drug penetration through the cornea in this study.

The epithelium, stroma, and endothelium of the cornea each have its own unique structure, which can represent a barrier for drug penetration into the eye [47]. The corneal epithelium's tight junctions and its lipophilic nature act as a barrier to hydrophilic drugs, and the stroma's highly hydrated structure limits the penetration of lipophilic drugs [47]. Any corneal disorganization caused by ultrasound application, which changes the structure of the first layer of the cornea and its tight junctions, is expected to enhance the drug penetration into the eye $[26,48]$. In comparison, drug penetration through the skin also increased as ultrasound application altered the stratum corneum and its lipid bilayer structure [32].

The in vivo study presented here is designed based on our previously performed in vitro study, which resulted in a statistically significant increase in the corneal permeability for dexamethasone sodium phosphate [26]. Results from our in vitro study showed a corneal permeability increase of 2.1 times at $400 \mathrm{kHz}$ and 1.5 times at $600 \mathrm{kHz}$, at intensity of $0.8 \mathrm{~W} / \mathrm{cm}^{2}$, as compared to sham-treated samples. Likewise, this in vivo study showed a statistically significant increase in drug delivery through the cornea. The increase in corneal permeability was greater in our in vivo study with maximum increase of 2.8 times, as compared with previous in vitro experiments [26]. In both studies, the level of damage observed in histology slides was limited to the epithelial layer, with no apparent damage in the stroma and endothelium [26].

The quantity of drug used was adequate to insure submerging of ultrasound transducer tip inside the solution. The maximum amount of drug used in the eye cup was approximately $15 \mathrm{~mL}$. There is no direct relationship between the quantity of drug used in our study and the quantity of drug delivered locally in eye drops. In our study, the eye was exposed to drug solution for $5 \mathrm{~min}$, which is less than the lag time (approximately $25 \mathrm{~min}$ ) allowing penetration of dexamethasone sodium phosphate through the cornea. The goal of this study was not only to compare the eye drops and the eye cup drug application but also to see if ultrasound can promote the drug delivery through the cornea in vivo.

In our previous in vitro studies, the spherical head diffusion cell was used to minimize the change to the natural shape of the eye, but this change in the eye structure as compared to in vivo situation may have been a contributing factor-likely minor to the enhancement of corneal permeability. Also, storing the eyes in nutrient-adjusted storage solution after the eyes are excised can cause deterioration of corneal barrier properties [49] which may increase corneal permeability in vitro in both ultrasoundtreated and sham-treated samples. In summary, the advantages of testing in vivo as compared to in vitro setup are the following: keeping the natural shape and structure of the rabbit eye, natural clearance mechanisms, perfusion, and the fact that eye does not potentially lose its barrier properties due to decay $[49,50]$.

Investigating the corneal permeability of dexamethasone sodium phosphate, using $400-$ and $600-\mathrm{kHz}$ ultrasound frequencies at $0.8 \mathrm{~W} / \mathrm{cm}^{2}$ for $5 \mathrm{~min}$, resulted in the enhancement of ocular drug delivery through the cornea, which was statistically significant as compared to shamtreated samples in in vitro and in vivo studies. There are differences in results from in vitro and in vivo studies that may be caused by experimental setup, environmental aspects, and tissue orientation. For example, the absorption of samples collected from receiver compartment of diffusion cells during in vitro study was measured using a spectrophotometer. The sensitivity of this device can have an impact on the amount of drug detected. On the other hand, sampling the actual aqueous humor from the eye in in vivo study and measuring the drug concentration inside this solution using chromatography methods can be more efficient. Moreover, all the samples in both cases were treated the same and were analyzed consistently.

In our current study, the major structural changes were observed in the epithelial layer of the cornea. Some of these changes included cell removal from the first layer of the epithelium. Also some epithelial cells from inner layers appeared lighter in color or their nucleus were missing. The stroma and endothelium were not damaged in most cases; however, detachment of endothelium was observed in some samples, which may be caused by processing artifacts [30,31]. Our current findings were consistent with our previous work where based on histological analysis, in both in vitro and in vivo corneal samples, the ultrasound-induced changes were observed mostly in the epithelial layers $[24,26,48]$. Results from our semi-quantitative analysis (as described in the 'Methods' section) showed that ultrasound-induced damage in the epithelial layers at frequency of $400 \mathrm{kHz}$ was four times higher $(p<0.01)$ in vivo $(n=6)$ and 1.8 times higher $(p<0.01)$ in vitro $(n=12)$ as compared to respective 
in vivo and in vitro sham-treated samples $(n=8-33)$. Using the same method, the damage in the epithelial layer after application of $600-\mathrm{kHz}$ ultrasound was three times higher $(p<0.05)$ in vivo $(n=6)$, and two times higher $(p<0.01)$ in vitro $(n=15)$ as compared to shamtreated samples $(n=8-33)$.

The in vitro corneas [26] are likely to have sustained more tissue damage than the in vivo corneas since the in vitro corneas were taken out the organism and handled. During in vivo study, the eyes were dissected after $90 \mathrm{~min}$ from the treatment and were considered as minimally damaged. However, during in vitro study, the eyes were shipped from a facility, which dissected the eyes approximately 20-24 h before we received them. There is a chance that these eyes were deteriorated while in the medium and during shipment. This factor may influence the differences between results in vitro and in vivo. By comparing in vivo and in vitro sham-treated corneas to the in vivo and in vitro corneas exposed to 400- and 600$\mathrm{kHz}$ ultrasound, it can be determined whether the tissue damage sustained by the experimental corneas is mainly due to the ultrasound exposure. The in vivo corneas usually had mostly intact epithelium, stroma, and endothelium in each of the samples, while all the in vitro corneas had large gaps in the epithelium and stroma and had fragmented or absent endothelium. Some of the in vivo corneas had more obvious tissue damage which was unlikely due to the ultrasound application. Some of the damages indicated above can be due to histological processing artifacts [30,31]. Our histological analysis indicated that exposing the corneas to $400-\mathrm{kHz}$ ultrasound affected the tissues of the corneas more significantly than exposure to $600-\mathrm{kHz}$ ultrasound, in both in vitro and in vivo cases. Results from semi-quantitative histological analysis for in vitro study showed that approximately two times increase $(p<0.01)$ in the epithelial damage was observed as compared to sham-treated samples $(n=33)$ using $400 \mathrm{kHz}(n=12)$ and $600 \mathrm{kHz}(n=15)$ ultrasound application at $0.8 \mathrm{~W} / \mathrm{cm}^{2}$ for $5 \mathrm{~min}$. Using the same method of analysis for the same parameters in vivo showed four times increase $(p<0.01)$ at frequency of $400 \mathrm{kHz}(n=6)$ and three times increase $(p<0.05)$ at frequency of $600 \mathrm{kHz}(n=6)$, as compared to shamtreated samples $(n=8)$. From the statistical results of layer thickness measurements, we learned that for $400-\mathrm{kHz}$ ultrasound application in vivo, the epithelium was significantly thinner $(p<0.001)$ as compared to control cases. The thickness changes in the stroma due to ultrasound application were not statistically significant. The thickness changes in the endothelium could not be analyzed with certainty since the endothelium was completely absent in some samples (and thus recorded as a value of 0 ), and this absence may have been due to histology processing artifacts as mentioned above.
As an ultrasound wave propagates through a medium, absorption and scattering cause the attenuation of ultrasound energy; transformation of ultrasound energy to heat is a result of this absorption [31,51]. The importance of thermal effects increases with an increase in frequency [34] and is also directly proportional to the ultrasound intensity and duty cycle [31]. Skin permeability and its diffusion coefficient were shown to be increased due to the increase of skin temperature resulting from ultrasound application [32]. In our current in vivo study, thermal effects of ultrasound leading to temperature increase in the cornea may have contributed to the increase in the corneal permeability. In this study, the maximum change in temperature was between $0^{\circ} \mathrm{C}$ and $6^{\circ} \mathrm{C}$, with average change of $3.2^{\circ} \mathrm{C} \pm 1.8^{\circ} \mathrm{C}$ for all treatment cases. The corneal permeability to water was shown previously to increase as temperature increased from $24^{\circ} \mathrm{C}$ to $34^{\circ} \mathrm{C}$ [52].

Excessive ultrasound energy can thermally and mechanically damage the eye; therefore, U.S. Food and Drug Administration (FDA) has enforced strict thermal and mechanical index limits for ophthalmic applications [53]. FDA guidelines permitted using only $I_{\text {SPTA.3 }}$ of $17 \mathrm{~mW} / \mathrm{cm}^{2}$ of acoustic energy for ocular application before Medical Device Act of 1976 was passed, which was later changed to $I_{\text {SPTA.3 }}$ of $50 \mathrm{~mW} / \mathrm{cm}^{2}[54,55]$. Thermal index (TI) is ratio of device's total acoustic power to the power needed to increase tissue's temperature by $1^{\circ} \mathrm{C}$ [53], and thermal index limit for ocular application is $\mathrm{TI}<1.0$ [55-57]. We believe that FDA regulations, in case of limits for intensities and temperature increase, are very restricted which may cause a great constraint on using the ultrasound in ocular drug delivery. More investigation using modeling and long-term survival studies is required to determine all potential adverse effects of ultrasound at our parameters in the eye.

The eye is vulnerable to thermal damage from ultrasound exposure because the absence of blood flow in the cornea and lens, both of which are avascular, results in slower cooling as the heat is absorbed by these tissues during ultrasound application [58,59]. Over-heating of the lens is a major concern because it may cause cataract formation; however, Guy et al. indicated that the potential risk would be generated if the temperature increases above $41^{\circ} \mathrm{C}$ [60]. Measuring the temperature of the lens was not feasible in our current study; therefore, further modeling and experimental investigations are needed to observe the impact of ultrasound application at our proposed parameters on potential heat generation in the lens.

One of the main goals for this study is to investigate the effectiveness of using ultrasound in ocular drug delivery and get some preliminary information regarding safety of ultrasound application. Indeed, future studies will focus on long-term survival experiments (up to 14 days) to determine adverse effects of ultrasound in different eye 
tissues. Further, lens can get overheated and be damaged as a result of thermal effects of ultrasound application. The hyperthermia level of temperature increase $\left(41^{\circ} \mathrm{C}-43^{\circ} \mathrm{C}\right)$ did not reach in our study. The maximum temperature increase during ultrasound application was $31^{\circ} \mathrm{C}$, which was below the hyperthermia level; therefore, we believed that the lens was not affected as a result of ultrasound application. The maximal corneal temperature in our experiments $\left(31^{\circ} \mathrm{C}\right)$ was below the normal physiological temperature of rabbit cornea of $34^{\circ} \mathrm{C}$ [61] due to the fact that the exposure to drug solution cooled down the cornea. The maximal corneal temperatures observed in this study were lower than the hyperthermia levels $\left(41^{\circ} \mathrm{C}-43^{\circ} \mathrm{C}\right)$; therefore, functional changes in the corneal epithelial cells due to heat were unlikely $[58,62]$. Modeling studies (that are currently ongoing) can be helpful addressing the changes in the lens, retina, and different tissues in the eye as a result of temperature increase during ultrasound application.

One of the key objectives of this study was to investigate the damages in the cornea due to ultrasound application. Putting a thermocouple inside the cornea would lead to errors in measurement of cornea permeability, which was another goal of this study, and would cause corneal damage, which interferes with the purpose of histology analysis of corneal damages. However, measuring temperature inside the cornea may be more accurate. A modeling study was completed that investigated the issue of temperature increase in different eye tissues.

In vivo study was designed to investigate the drug delivery inside the eye while it is intact and inside the body. Also dissecting the eye right after the experiment keeps it fresh which decreases the chance of deterioration. The greater intraocular pressure causes decrease in scleral and corneal permeability. Rudnick et al. indicated that the scleral permeability to small compounds is a weak function of intraocular pressure and mostly depends on molecular weight [63]. However, we did not measure the intraocular pressure and its effect on drug penetration through the cornea. Several animal studies indicated that using HIFU could be a possible and effective technique to reduce intraocular pressure [3]. Ultrasound has the potential for intraocular pressure reduction, which in our case can have an effect on increasing ocular drug permeability; however, this effect in not expected to be major based on previous studies.

The same anesthesia method was performed in both ultrasound treatment and sham treatment cases, so its effects should not impact the study outcome that ultrasound application leads to increase the drug delivery through the cornea. Usually, the depressants of the central nervous system using general anesthetics will decrease intraocular pressure in relation with the depth of anesthesia, and the type of drug can also be a factor effecting the change in intraocular pressure. However, ketamine causes rising on intraocular pressure that is less than the decrease caused by other general anesthesia [64]. There is a possibility that anesthesia can affect drug penetration through the cornea, but this was not investigated in our studies. The sham treatment underwent the same anesthesia methods as ultrasound treatment, which allowed us to observe the effects of ultrasound specifically.

The topical administration of a drug through the cornea, especially for hydrophilic drugs, is a challenging task. Less than $5 \%$ of the applied drug can penetrate through the cornea because of the eye barriers $[9,11,12]$; for example, the epithelium layer has lipophilic properties, which makes it hard for the hydrophilic drug to penetrate into the eye. In other words, we can state that both points made here are important. Enhancement in the delivery of dexamethasone sodium phosphate by $2-3$ times is considered clinically significant because this drug has hydrophilic properties and its penetration through the cornea is a challenge.

Even though the results from our in vivo study did not show the temperature increase at the hyperthermia level $\left(41^{\circ} \mathrm{C}-43^{\circ} \mathrm{C}\right.$ ), a modeling study (currently in progress) was designed to investigate the temperature changes in the lens, retina, and different tissues in the eye. The results from this modeling study (unpublished data) indicate that the potential temperature increase was minimal and lower than the hyperthermia level in all the eye tissues using ultrasound application at parameters used in in vivo study.

\section{Conclusions}

Ultrasound may be used as an effective tool to enhance ocular drug delivery. This study explored the feasibility of using ultrasound for delivery of a clinically relevant drug, dexamethasone sodium phosphate, in an in vivo rabbit model. This study was designed using parameters based on the results from our previously performed in vitro studies. Increase of 2.8 times $(p<0.05)$ and 2.4 times $(p<0.05)$ in the drug penetration through the cornea was observed using ultrasound at 400 and $600 \mathrm{kHz}$, respectively. Minor damage and structural changes were present in the corneal epithelium of the ultrasoundtreated corneas. Further studies are needed to fully investigate the safety aspects of ultrasound application in ocular drug delivery such as long-term monitoring of recovery of corneal barrier properties and safety of ultrasound exposure in different eye tissues including the lens and retina. It would also be of potential clinical interest to perform studies involving ultrasound application for the enhancement of delivery of anti-fungal and anti-viral ocular drugs currently used in treatment of different eye diseases such as keratitis, scleritis, and herpetic eye disease. 


\section{Competing interests}

The authors declare that they have no competing interests.

\section{Authors' contributions}

MN designed the experimental setup, acquired data, performed all acoustic measurements, analyzed histology slides, and drafted/edited the manuscript with a great help from Dr. VZ. AS helped with analyzing histology slides and statistical analysis and edited the manuscript. SC designed some parts of the eye cup, helped with data collection and animal handling during the experiment, and edited the manuscript. Drs. SM, JL, and CG are our collaborators from GWU Ophthalmology Department and provided knowledge and medical expertise about ophthalmology and ensuring safety of the eye. Dr. VZ supervised MN, AS, and SC in experimental research, monitored all parts of the project, initiated the study, provided the concept and design, and revised/edited the manuscript. All authors read and approved the final manuscript.

\section{Acknowledgements}

This work was supported by National Eye Institute grant NIH5R21EY01873702. We thank Dr. Shutao Wang for his help during the experiments

\section{Author details}

${ }^{1}$ Department of Electrical and Computer Engineering, George Washington University, Washington, DC 20052, USA. ²Department of Ophthalmology, George Washington University, Washington, DC 20052, USA.

Received: 9 November 2013 Accepted: 4 February 2014 Published: 31 March 2014

\section{References}

1. Mundt $\mathrm{GH}$, Hugues WE. Ultrasonics in ocular diagnosis. Am J Ophthalmol. 1956; 41:488-98.

2. Allemann N, Silverman RH, Reinstein DZ, Coleman DJ. High-frequency ultrasound imaging and spectral analysis in traumatic hyphema. Ophthalmology. 1993; 100:1351-7.

3. Aptel F, Lafon C. Therapeutic applications of ultrasound in ophthalmology. Int J Hyperthermia. 2012; 28:405-18. doi:10.3109/02656736.2012.665566.

4. Sterk CC, vd Valk PH, van Hees CL, van Delft JL, van Best JA, Oosterhuis JA. The effect of therapeutic ultrasound on the average of multiple intraocular pressures throughout the day in therapy-resistant glaucoma. Graefes Arch Clin Exp Ophthalmol. 1989; 227(1):36-8.

5. Silverman $\mathrm{RH}$, Vogelsang $B$, Rondeau MJ, Coleman DJ. Therapeutic ultrasound for the treatment of glaucoma. Am J Ophthalmol. 1991; 111(3):327-37.

6. Aptel F, Charrel T, Lafon C, Romano F, Chapelon JY, Blumen-Ohana E, Nordmann JP, Denis P. Miniaturized high-intensity focused ultrasound device in patients with glaucoma: a clinical pilot study. Invest Ophthalmol Vis Sci. 2011; 52:8747-53. doi:10.1167/iovs.11-8137.

7. Sonoda S, Tachibana K, Uchino E, Okubo A, Yamamoto M, Sakoda K, Hisatomi T, Sonoda KH, Negishi Y, Izumi Y, Takao S, Sakamoto T. Gene transfer to corneal epithelium and keratocytes mediated by ultrasound with microbubbles. Invest Ophthalmol Vis Sci. 2006; 47(2):558-64.

8. Park J, Zhang Y, Vykhodtseva N, Akula JD, McDannold NJ. Targeted and reversible blood-retinal barrier disruption via focused ultrasound and microbubbles. PloS One. 2012:1932-6203. doi:10.1371/journal.pone. 0042754.

9. Eljarrat-Binstock E, Pe'er J, Domb AJ. New techniques for drug delivery to the posterior eye segment. Pharm Res. 2010; 27:530-43. doi:10.1007/s11095-0090042-9.

10. Sasaki H, Igarashi Y, Nagano T, Nishida K, Nakamura J. Different effects of absorption promoters on corneal and conjunctival penetration of ophthalmic beta-blockers. Pharm Res. 1995; 12(8):1146-50.

11. Achouri $D$, Alhanout $K$, Piccerelle $P$, Andrieu V. Recent advances in ocular drug delivery. Drug Dev Ind Pharm. 2012:1-19. doi:10.3109/03639045. 2012.736515.

12. Gaudana R, Ananthula HK, Parenky A, Mitra AK. Ocular drug delivery. AAPS J. 2010; 12:348-60. doi:10.1208/s12248-010-9183-3.

13. Kompella UB, Kadam RS, Lee VH. Recent advances in ophthalmic drug delivery. Ther Deliv. 2010; 1:435-56. doi:10.4155/TDE.10.40.
14. Ke TL, Clark AF, Gracy RW. Age-related permeability changes in rabbit corneas. J Ocul Pharmacol Ther. 1999; 15:513-23.

15. Robinson JR. Ocular anatomy and physiology relevant to ocular drug delivery. In: Mitra AK, editor. Ophthalmic Drug Delivery Systems. New York: Marcel Dekker; 1993: p. 1929-57.

16. Hu X, Hao L, Wang H, Yang X, Zhang G, Wang G, Zhang X. Hydrogel contact lens for extended delivery of ophthalmic drugs. Int J Polymer Science. 2011:1-9. doi:10.1155/2011/814163.

17. Mishra GP, Bagui M, Tamboli V, Mitra AK. Recent applications of liposomes in ophthalmic drug delivery. Journal of Drug Delivery. 2011:1-14. doi:10.1155/2011/863734.

18. Cohen AE, Assang C, Patane MA, From S, Korenfield M. Evaluation of dexamethasone phosphate delivered by ocular iontophoresis for treating noninfectious anterior uveitis. Ophthalmology. 2012; 119:66-73. doi:10.1016/j.ophtha.2011.07.006.

19. Eljarrat-Binstock E, Raiskup F, Stepensky D, Domb AJ, Frucht-Pery J. Delivery of gentamicin to the rabbit eye by drug-loaded hydrogel iontophoresis. Invest Ophthalmol Vis Sci. 2004; 45:2543-8.

20. Lam TT, Fu J, Chu R, Stojack K, Siew E, Tso MOM. Intravitreal delivery of ganciclovir in rabbits by transscleral iontophoresis. J Ocul Pharmacol. 1994; 10:571-5.

21. Yasukawa T, Ogura Y, Kimura H, Sakurai E, Tabata Y. Drug delivery from ocular implants. Expert Opin Drug Deliv. 2006; 3(2):261-3.

22. Du J, Shi QS, Sun Y, Lui PF, Zhu MJ, Du LF, Duan YR. Enhanced delivery of monomethoxypoly (ethylene glycol)-poly(lactic-co-glycolic acid)-poly I-lysine nanoparticles loading platelet-derived growth factor BB small interfering RNA by ultrasound and/or microbubbles to rat retinal pigment epithelium cells. J Gene Med. 2011:312-23. doi:10.1002/jgm.1574.

23. Hiscott $P$, Armstrong D, Batterbury M, Kaye S. Repair in avascular tissue: fibrosis in the transparent structures of the eye and thrombospondin 1 Histol Histopathol. 1999; 14:1309-20.

24. Zderic V, Clark J, Vaezy S. Drug delivery into the eye with the use of ultrasound. J Ultrasound Med. 2004; 23:1349-59.

25. Nuritdinov VA. Phonophoresis and cavitation [in Russian]. Vestn Oftalmol. 1981:56-8

26. Nabili M, Patel H, Mahesh SP, Liu J, Geist C, Zderic V. Ultrasound-enhanced delivery of antibiotics and anti-inflammatory drugs into the eye. Ultrasound Med Biol. 2013; 39(4):638-46. doi:10.1016/j.ultrasmedbio.2012.11.010.

27. Gwon A. The rabbit in cataract/IOL surgery. In: Panagiotis A, editor. Animal Models in Eye Research. San Diego: Elsevier; 2008: p. 184-99.

28. Cheung ACY, Yu Y, Tay D, Wong HS, Ellis-Behnke R, Chau Y. Ultrasound enhanced intrascleral delivery of protein. Int J Pharm. 2010; 401:16-24. doi:10.1016/j.jpharm.2010.09.001.

29. Christensen DA. Ultrasonic Bioinstrumentation. 1st ed. Hoboken: Wiley; 1988.

30. Silverman RH, Lizzi FL, Ursea BG, Cozzarelli L, Ketterling JA, Deng CX, Folberg R, Coleman DJ. Safety levels for exposure of cornea and lens to very high-frequency ultrasound. J Ultrasound Med. 2001; 20(9):979-86.

31. Polat BE, Hart D, Langer R, Blankschtein D. Ultrasound-mediated transdermal drug delivery: mechanisms, scope, and emerging trends. J Control Release. 2011; 152:330-48. doi:10.1016/j.jconrel.2011.01.006.

32. Mitragotri S, Edwards D, Blankschtein D, Langer R. A mechanistic study of ultrasound-enhanced transdermal drug delivery. J Pharm Sci. 1995; 84:697-706.

33. Paliwal S, Mitragotri S. Ultrasound-induced cavitation: applications in drug and gene delivery. Expert Opin Drug Deliv. 2006; 3(6):713-26.

34. Ahmadi F, McLoughlin IV, Chauhan S, terHaar G. Bio-effects and safety of low-intensity, low-frequency ultrasonic exposure. Prog Biophys Mol Biol. 2012; 108:119-38.

35. Dijkmans PA, Juffermans LJ, Musters RJ, van Wamel A, ten Cate FJ, van Gilst W, Visser CA, de Jong N, Kamp O. Microbubbles and ultrasound: from diagnosis to therapy. Eur J Echocardiogr. 2004; 5:245-56.

36. Tang H, Wang CC, Blankschtein D, Langer R. An investigation of the role of cavitation in low-frequency ultrasound-mediated transdermal drug transport. Pharm Res. 2002; 19(8):1160-9.

37. Miller DL. A review of the ultrasonic bioeffects of microsonation, gas-body activation, and related cavitation-like phenomena. Ultrasound Med Biol. 1987; 13:443-70

38. Postema M, Gilja OH. Ultrasound-directed drug delivery. Curr Pharm Biotechnol. 2007; 8(6):355-61.

39. Peach R, Gompf B. Microimplosions: cavitation collapse and shock wave emission on a nanosecond time scale. Phys Rev Lett. 2000; 84(6):1328-30. 
40. Martin RW, Hwang JH. Therapeutic potential and consideration of high intensity ultrasound in gastroenterology. In: Odegaard S, Gilja $\mathrm{OH}$ Gregersen H, editors. Basic and New Aspects of Gastrointestinal Ultrasonography, Advanced Series in Biomechanics. 2005th ed. Singapore: World Scientific; 2005: p. 211-42.

41. Saroha K, Sharma B, Yadav B. Sonophoresis: an advanced tool in transdermal drug delivery system. Int J Curr Pharm Res. 2011; 3(3):89-97.

42. Nyborg WL. Physical Mechanisms for Biological Effects of Ultrasound. Department of Health, Education, and Welfare. Washington, DC: U.S. FDA; 1978: p. 78-8062.

43. American Institute of Ultrasound in Medicine (AIUM) Report. Section 2-definition and description of non-thermal mechanisms. J Ultrasound Med. 2000; 19:77-84.

44. National Council on Radiation Protection and Measurements (NCRP). Biological effects of ultrasound: mechanical and clinical implications. Recommendations of the National Council on Radiation Protection and Measurements, NCRP Report No.74. Bethesda; 1983: p. 84-6.

45. Williams AR. Ultrasound: Biological Effects and Potential Hazards. London: Academic: 1983

46. Tsivgoulis G, Alexandrov A. Ultrasound-enhanced thrombolysis in acute ischemic stroke: potential, failures, and safety. Neurotherapeutics. 2007; 4(3):420-7.

47. Fischbarg J. The corneal endothelium. In: Fischbarg J, editor. The Biology of Eye. New York: Academic; 2006: p. 113-25.

48. Zderic V, Clark Jl, Martin RW, Vaezy S. Ultrasound-enhanced transcorneal drug delivery. Cornea. 2004; 23:804-11.

49. Doughty MJ. Evaluation of the effects of saline versus bicarbonatecontaining mixed salts solutions on rabbit corneal epithelium in vitro. Ophthalmic Physiol Opt. 1995; 15:585-99.

50. Jester JV, Petroll WM, Garana RM, Lemp MA, Cavanagh HD. Comparison of in vivo and ex vivo cellular structure in rabbit eyes detected by tandem scanning microscopy. J Microsc. 1992; 165(Pt 1):169-81.

51. Ensminger D, Stulen FB. Ultrasonic: Data, Equations, and their Practical Uses. Boca Raton: CRC: Taylor \& Francis Group; 2008.

52. Green K, Downs SJ. Corneal membrane water permeability as a function of temperature. Invest Ophthalmol. 1976; 15:304-7.

53. Palte HD, Gayer S, Arrieta E, Scot Shaw E, Nose I, Lee E, Arheart KL, Dubovy S, Birnbach DJ, Parel JM. Are ultrasound-guided ophthalmic blocks injurious to the eye? A comparative rabbit model study of two ultrasound devices evaluating intraorbital thermal and structural changes. Anesth Analg. 2012; 115(1):194-201. doi:10.1213/ANE.0b013e318253622e.

54. Herman BL, Harris GR. Theoretical study of steady-state temperature rise within the eye due to ultrasound insonation. IEEE Trans Ultrason Ferroelectr Frea Control. 1999; 46:1566-74.

55. Food and Drug Administration (FDA). Guidance for Industry and FDA Staff Information for Manufacturers Seeking Marketing Clearance of Diagnostic Ultrasound Systems and Transducers. Silver Spring: U.S. FDA; 2008.

56. Barnett SB. World Federation for Ultrasound in Medicine and Biology (WFUMB) Symposium on safety of ultrasound in medicine: conclusions and recommendations on thermal and non-thermal mechanisms for biological effects of ultrasound. Ultrasound Med Biol. 1998; 24:1-55.

57. Safety Group of the British Medical Ultrasound Society (BMUS). Guidelines for the safe use of diagnostic ultrasound equipment. Ultrasound. 2010; 18:52-9.

58. Cucevic V, Brown AS, Foster FS. Thermal assessment of $40 \mathrm{MHz}$ pulsed Doppler ultrasound in human eye. Ultrasound Med Biol. 2005; 31:565-73.

59. Kowalczuk L, Boudinet M, El Sanharawi M, Touchard E, Naud MC, Saïed A, Jeanny JC, Behar-Cohen F, Laugier P. In vivo gene transfer into the ocular ciliary muscle mediated by ultrasound and microbubbles. Ultrasound Med Biol. 2011; 37:1814-27. doi:10.1016/j.ultrasmedbio.2011.07.010

60. Guy AW, Lin JC, Kramar PO, Emery AF. Effect of 2450-Mhz radiation on the rabbit eye. IEEE Trans Microw Theory Techn. 1975; 23:492-8. doi:10.1109/ TMTT.1975.1128606.

61. Efron N, Young G, Brennan NA. Ocular surface temperature. Curr Eye Res. 1989; 8:901-6
62. Yamaguchi K, Barbe MF, Brown IR, Tytell M. Induction of stress (heat shock) protein 70 and its mRNA in rat corneal epithelium by hyperthermia. Curr Eye Res. 1990; 9(9):913-8.

63. Rudnick DV, Noonan JS, Geroski DH, Prausnitz MR, Edelhauser HF. The effect of intraocular pressure on human and rabbit scleral permeability. IOVS. 1999: 40(12):3054-8.

64. Duncalf D. Anesthesia and intraocular pressure. Bull N Y Acad Med. 1975; 51(3):374-81.

doi:10.1186/2050-5736-2-6

Cite this article as: Nabili et al:: Ultrasound-enhanced ocular delivery of dexamethasone sodium phosphate: an in vivo study. Journal of

Therapeutic Ultrasound 2014 2:6.

\section{Submit your next manuscript to BioMed Central and take full advantage of:}

- Convenient online submission

- Thorough peer review

- No space constraints or color figure charges

- Immediate publication on acceptance

- Inclusion in PubMed, CAS, Scopus and Google Scholar

- Research which is freely available for redistribution

Submit your manuscript at www.biomedcentral.com/submit
C Biomed Central 